\section{Repeated transcranial magnetic stimulation effect over treatment- resistant auditory hallucinations in a female patient with schizophrenia}

\section{Efeito da estimulação maǵnética transcraniana repetida sobre alucinações auditivas refratárias em uma paciente com esquizofrenia}

\section{Dear Editor,}

Treatment-resistant auditory hallucinations (TRAH) occur in $20 \%$ of treated schizophrenia patients and contribute to disability and morbidity. Repeated transcranial magnetic stimulation (rTMS) is a recent strategy for TRAH which has been tested in around 20 randomized controlled trials, and found controversial results. Hoffmann et al. reported data from 50 schizophrenia/schizoaffective disorder patients showing superiority of rTMS over sham stimulation in auditory hallucinations with a 9-day, $1-\mathrm{Hz}$ stimulation in the left temporo-parietal cortex (LTPC), using the Auditory Hallucination Hoffmann Scale (AHS) and the Clinical Global Impression Scale (CGI) as assessment instruments. ${ }^{1}$ Similar studies evidenced conflicting results ${ }^{2-4}$ putatively due to differences in stimulation parameters, such as length, number, potency, interval, and brain side. Session number was important in treatment - resistant depression, with clear superiority of 20-session over 10-session protocols. ${ }^{5}$ Additionally, in the studies reviewed, all interventions were provided for short time span, with no randomized controlled trials providing long-duration rTMS and prolonged follow-up.

We describe here one case of a patient with TRAH who was submitted to a long-duration rTMS (20 days), followed by initial remission and further relapse after 10 months, although with a significant improvement after repeated intervention.
A 59 year-old female patient, who had her illness onset at age 26 , presented to treatment with persistent and severe auditory hallucinations and persecutory delusions. Over 33 years she was continuously treated with several typical and atypical antipsychotics at full therapeutic dosages and for sufficient time, having had slight improvement of overall status, maintaining severe hallucinations and delusions and related social and professional disability and impairment. After 11 unilateral electroconvulsive therapy sessions her Brief Psychiatric Rating Scale (BPRS) scores dropped from 33 to 22, and her Mini-Mental State Examination (MMSE) dropped from 28 to 19. However, the treatment was interrupted due to cognitive side effects. She was maintained on clozapine $800 \mathrm{mg} /$ day, amisulpride 600 mg/day and lamotrigine $400 \mathrm{mg} /$ day for one year, with limited effect over social activities and persecutory delusions, maintaining severe auditory hallucinations ( $\mathrm{AHS}=30$ ).

In early 2005 she received twenty 20 -minute sessions of $1-\mathrm{Hz}$ LTPC rTMS, at $90 \%$ motor threshold with a clinically relevant $50 \%$ AHS reduction. The largest effects were in auditory hallucinations frequency and intensity. Patient, relatives and Care Provider reported global improvement in social and family interaction and self-care.

One year after the first treatment, the patient reported recurrence of the auditory hallucinations and received the same treatment protocol with significant symptom improvement, having AHS scores dropped from 24 to 17 and BPRS scores from 43 to 15 , without cognitive deficits (MMSE $=28$ ). Patient and relatives reported marked improvement in spontaneity, social skills, and family relations. These findings not only replicate previous studies, but also suggest that patient's outcomes goes beyond symptom relief, including reduction of negative symptoms and improvement in quality of life. We also suggest that large-duration protocols may be better, and that effects may last about 6-8 months, being the treatment repetition capable to induce similar effects, without the development of tolerance. These findings require replication in larger samples using sham-controlled double-blind procedures, and with the assessment of additional outcomes, such as quality of life and affect regulation.

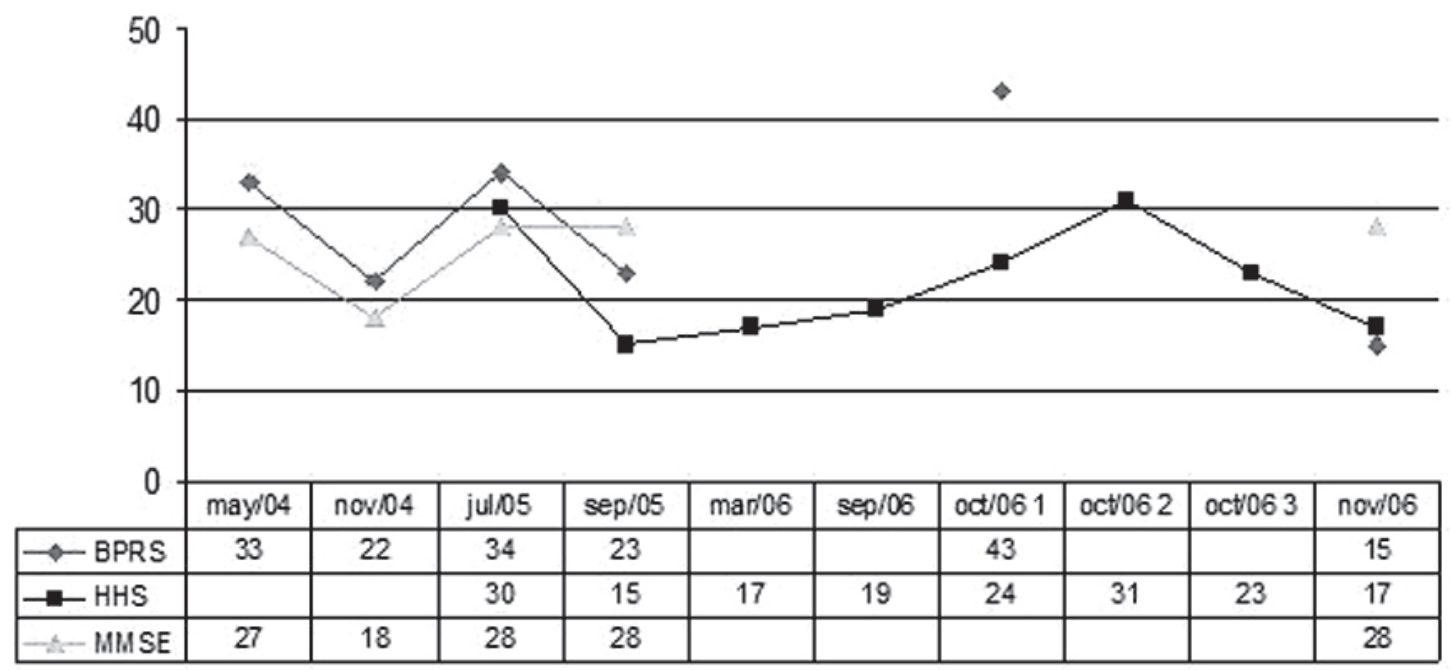

Figure 1 - Psychopathology evolution

BPRS = Brief Psychiatric Rating Scale

AHS = Hoffmann Hallucination Scale

MMSE $=$ Mini-Mental State Examination 
Gabriela Favalli

Schizophrenia and Dementia Program (PRODESQ), Hospital das Clínicas de Porto Alegre (HCPA), Universidade Federal do Rio Grande do Sul (UFRGS), Porto Alegre (RS), Brazil

Alexei Gil

Post-Graduate Program in Medical Sciences, Universidade Federal do Rio Grande do Sul (UFRGS), Porto Alegre (RS), Brazil

Schizophrenia and Dementia Program (PRODESQ), Hospital de Clínicas de Porto Alegre (HCPA), Universidade Federal do Rio Grande do Sul (UFRGS), Porto Alegre (RS), Brazil

Maria Inês Lobato Post-Graduate Program in Medical Sciences, Universidade Federal do Rio Grande do Sul (UFRGS), Porto Alegre (RS), Brazil Schizophrenia and Dementia Program (PRODESQ), Hospital de Clínicas de Porto Alegre (HCPA), Universidade Federal do Rio Grande do Sul (UFRGS), Porto Alegre (RS), Brazil

Marco Antônio Marcolin Transcranial Magnetic Stimulation Group, Psychiatry Institute, Hospital das Clínicas, Universidade de São Paulo (USP), São Paulo (SP), Brazil

Paulo Belmonte-de-Abreu Post-Graduate Program in Medical Sciences, Universidade Federal do Rio Grande do Sul (UFRGS), Porto Alegre (RS), Brazil Department of Psychiatry, Medical School, Universidade Federal do Rio Grande do Sul (UFRGS), Porto Alegre (RS), Brazil

Financing: None

Conflict of interests: None

\section{References}

1. Hoffman RE, Gueorguieva R, Hawkins KA, Varanko M, Boutros NN, Wu YT, Carrol K, Krystal JH. Temporoparietal transcranial magnetic stimulation for auditory hallucinations: safety, efficacy and moderators in a fifty patient sample. Biol Psychiatry. 2005;58(2):97-104.

2. Poulet E, Brunelin J, Bediou B, Bation R, Forgeard L, Dalery J, d'Amato T, Saoud M. Slow transcranial magnetic stimulation can rapidly reduce resistant auditory hallucinations in schizophrenia. Biol Psychiatry. 2005;57(2):188-91.

3. Rosa MO, Rosa MA, Rigonatti MC, Cabral SB, Myczkowski ML, Sartorelli MC, Tavares H, Rumi DO, Marcolin MA. Effects of repetitive trancranial magnetic stimulation on auditory hallucinations refractory to clozapine. J ECT. 2006;22(1):79.

4. Fitzgerald PB, Benitez J, Daskalakis, JZ, Brown TL, Marston NA, de Castela A, Kulkami J. A double blind sham controlled trial of repetitive transcranial magnetic stimulation in the treatment of refractory auditory hallucinations. J Clin Psychopharmacol. 2005;25(4):358-62.

5. Fitzgerald PB, Brown TL, Marston NA, de Castella A, Daskalakis ZJ, Kulkami J. Transcranial magnetic stimulation in the treatment of depression: a double-blind, placebo-controlled trial. Arch Gen Psychiatry. 2003;60(10):1002-8.

\section{Refractory schizophrenia: a neglected clinical problem}

\author{
Esquizofrenia refratária: um problema \\ clínico negligenciado
}

Dear Editor,

Treatment refractoriness is an important and frequent problem among individuals with schizophrenia, causing enormous suffering and distress to patients and families. ${ }^{1,2}$ Although psychosocial interventions are fundamental, antipsychotic treatment is the mainstay of care for schizophrenia. However, about $30 \%$ of the schizophrenic patients do not respond adequately to adequate antipsychotic treatment. ${ }^{2}$ Treatment of refractory schizophrenia criteria were established based on Kane's studies with clozapine and modified by the IPAP algorithm: ${ }^{3} 1$ ) no period of good functioning in the previous 5 years; 2) prior non-response to at least 2 antipsychotic drugs of two different chemical classes for at least 4-6 weeks each at doses $\geqslant 400 \mathrm{mg}$ equivalents of chlorpromazine or $5 \mathrm{mg} /$ day of risperidone; 3) moderate to severe psychopathology, especially positive symptoms. Several studies and metanalyses confirm clozapine's superior efficacy on treatment-refractory schizophrenia, ${ }^{2,3}$ and the psychopharmachologic mechanisms involved in this superiority are not clear yet. ${ }^{2,4}$ Clozapine is the best validated approach, but there are several other interesting approaches being developed. ${ }^{5}$ Although refractory schizophrenia represents a challenge to the clinicians, it is often underdiagnosed and undertreated.

We have performed a study investigating the detection of the refractory schizophrenia cases in an academic outpatient clinic (Schizophrenia Program - PROESQ). We have developed a screening questionnaire describing the clinical characteristics, previous treatments and possible causes of refractoriness, and assessed 198 outpatients with diagnosis of schizophrenia and schizoaffective disorder. All psychiatrists $(n=17)$ were asked to point out their possibly refractory outpatients, based on four criteria stated in the questionnaire: treatment non-responder, non-compliant, clozapine user, and concomitant use of two or more antipsychotic drugs. Seventy patients (35\%) met criteria for treatment refractory schizophrenia after extensive clinical chart review. Forty-seven patients (67\%) were males, mean age 36 y.o. (range 17-59), the age of onset of schizophrenia was 22 y.o. (range 12-44), disease duration 13.7 years $(S D=7.8)$, the number of psychiatric hospitalizations was 1.8 ( 0 to $10, S D=2.1$ ), and $34.3 \%$ were alcohol and/or drug abusers/dependents. The treatment-refractory patients had used on average $4.6(\mathrm{SD}=1.7)$ antipsychotic drugs, however only $43 \%$ ( $15 \%$ of all patients) had used clozapine.

These results show a low rate of recognition and appropriate treatment of refractory schizophrenia patients in an academic service in Brazil. We presume that these rates are even lower in other centers in Brazil, being a large proportion of the psychiatrists tolerant to imperative residual symptoms and misdiagnosing clinical refractoriness. Improvement on current psychiatric training is necessary to educate clinicians to recognize treatment-refractory schizophrenia and to manage the related clinical issues, such as poor compliance to medication, alcohol and drug abuse/ dependence, and comorbid depression. To properly treat refractory schizophrenia patients in the public sector in Brazil, the outpatient 